\title{
Annihilation of singlet fermionic dark matter into two photons
}

\author{
M.M. Ettefaghi ${ }^{1}$ and R. Moazzemi ${ }^{2}$ \\ Department of Physics, The University of Qom, Qom 371614-611, Iran.
}

\begin{abstract}
We consider an extension of the standard model in which a singlet fermionic particle, to serve as cold dark matter, and a singlet Higgs are added. We perform a reanalysis on the free parameters. In particular, demanding a correct relic abundance of dark matter, we derive and plot the coupling of the singlet fermion with the singlet Higgs, $g_{s}$, versus the dark matter mass. We analytically compute the pair annihilation cross section of singlet fermionic dark matter into two photons. The thermally averaged of this cross section is calculated for wide range of energies and plotted versus dark matter mass using $g_{s}$ consistent with the relic abundance condition. We also compare our results with the Fermi-Lat observations.
\end{abstract}

\section{PACS:}

\footnotetext{
${ }^{1}$ mettefaghi@qom.ac.ir
}

${ }^{2}$ r.moazzemi@qom.ac.ir 


\section{Introduction}

Although there exist numerous cosmological and astrophysical indications which confirm the presence of the non-baryonic dark matter, the nature of this substance remains unknown $[1,2]$. One of the most important motivations for the extension of the standard model (SM) is to introduce an appropriate candidate for dark matter. Among the various candidates proposed by the theories beyond the SM, weakly interacting massive particles (WIMP) are the most popular one. For instance supersymmetry models with $\mathrm{R}$ parity $[1,2]$, the extra dimensional models with conserved Kaluza-Klein (KK) parity [3], the T-parity conserved little Higgs model [4], and so on provide a WIMP dark matter candidate. Meanwhile, these models introduce others degrees of freedom besides of the dark matter which have not been confirmed experimentally until now. Of course, in addition to the dark matter there exist others motivations such as neutrino oscillation, baryon asymmetry, hierarchy problem, and so on for considering such models. However, maybe it seems natural to consider the most economical model for the explanation of these anomalies. For instance, if we want to explain dark matter anomaly, adding a singlet scalar to the SM content to play the dark matter role is the most minimal model. Several authors have studied the singlet scalar dark matter [5]. Alternatively, adding a singlet fermion together with a singlet Higgs to the SM content leads to a renormalizable theory for explanation of the dark matter [6]. Losing the renormalizablity of the theory we can have more minimal model with singlet fermionic dark matter (SFDM) [7, 8]. In all above scenarios, the weak interactions of WIMPs are the main key for explaining the thermal production of them in the early universe (for review see [1,2]). Additionally, these weak interactions can provide an opportunity for search dark matter candidates through their production in high energy accelerators [9], their direct detection [10], and their indirect detection i.e. astrophysical observations of the annihilation or decay products of the dark matter in our galaxy or beyond.

In fact, the dark matter anomaly is a mutual problem between particle physics and astrophysics; on one hand, we need to know particle physical properties of dark matter to attribute a galaxy distribution to it and on the other hand we need to know the distribution of the dark matter to construe a direct or indirect detected signals as dark matter signals.

In the WIMP scenario, the weak interaction of dark matter would produce observable SM particles, such as charged anti-matter particles, photons and neutrinos. Among these, neutrinos and photons have advantage in comparison to others because they

keep their source information during the streaming. Moreover, the very small cross 
sections of the neutrinos make their flux very difficult to detect. Therefore, the gamma ray signature of the dark matter has been investigated extensively (For review see [11] and references therein). Since the continuum gamma ray emission from dark matter annihilation could be confused with astrophysical backgrounds, e.g. emission from galactic cosmic rays or millisecond pulsars, the study of the monochromatic gamma ray is important. Monochromatic gamma ray signatures have been studied for some dark matter candidates in the literature [12]. In this paper we investigate monochromatic gamma ray in the context of the SFDM model.

The most minimal model, which includes a singlet fermion to serve as dark matter, is discussed in [7]. In this model the leading interaction between dark matter and the SM particle is given by the dimension-five term $\frac{1}{\Lambda} H^{\dagger} H \bar{\psi} \psi$. Here $H, \psi$ and $\Lambda$ are the SM Higgs doublet, the dark matter fermion and a new physics scale, respectively. Moreover, it is possible to explain the dark matter production in the early universe by a renormalizable theory if the Higgs sector of the SM is extended by a new singlet Higgs [6]. Also in the noncommutative space-time we can have a singlet fermionic dark matter which is involved in the noncommutative induced $U(1)$ gauge theory [8]. There are also some others phenomenological studies on this model [13].

In this paper, we reobtain the thermal average of the annihilation of SFDM into the SM particles. Then, demanding the correct relic abundance we derive the coupling of SFDM with the singlet Higgs versus dark matter mass. For this calculation we use a set of Higgs masses so that the recent CMS and ATLAS results are respected ${ }^{3}$. Then we obtain the cross section of the annihilation of SFDM pairs into two photons. The thermal average of this cross section is calculated throughout a favorite WIMP mass interval including resonance region. We depict the SFDM annihilation cross section into two photons versus dark matter mass, $m$, using $g_{s}$ consistent with relic abundance condition. We have organized the paper as follows: In the next section, we review the renormalizable extension of the SM by a singlet fermion which can be served as a cold dark matter and derive the $g_{s}$ consistent with the relic density abundance. In Section 3, we calculate the two photons annihilation of the singlet fermion pairs and its thermally averaged cross section. We summarize our result in the last section.

\footnotetext{
${ }^{3}$ http://cms.web.cern.ch/news/observation-new-particle-mass-125-gev
} 


\section{Renormalizable Extension of the Standard Model by a Singlet Fermion}

It is possible to consider a singlet Dirac fermion beyond the SM which can serve as cold dark matter. The lepton number and baryon number of this new fermion are taken to be zero. Hence, there is not any mixing between the new fermion and the SM fermions. If a singlet Higgs is added to this model, the interactions between the singlet fermion and SM particles can be explained by a renormalizable manner. Explicitly, the singlet fermion and singlet Higgs are coupled as follows:

$$
\mathcal{L}_{\text {int }}=g_{s} \bar{\psi} \psi S
$$

and the singlet Higgs can be coupled with the SM Higgs through the following terms:

$$
\mathcal{L}_{\mathrm{SH}}=-\lambda_{1} H^{\dagger} H S-\lambda_{2} H^{\dagger} H S^{2}
$$

Therefore, in addition to the above terms, we need to add the following terms for singlet fermion and singlet Higgs to the SM Lagrangian:

$$
\begin{gathered}
\mathcal{L}_{\psi}=\bar{\psi}\left(i \not \partial-m_{\psi}\right) \psi \\
\mathcal{L}_{\mathrm{S}}=\frac{1}{2}\left(\partial_{\mu} S\right)\left(\partial^{\mu} S\right)-\frac{m_{0}^{2}}{2} S^{2}-\frac{\lambda_{3}}{3 !} S^{3}-\frac{\lambda_{4}}{4 !} S^{4} .
\end{gathered}
$$

The scalar potential given in (2) and (4) together with the SM potential, $-\mu^{2} H^{\dagger} H+$ $\lambda_{0}\left(H^{\dagger} H\right)^{2}$, lead to the vacuum expectation values

$$
\langle H\rangle=\frac{1}{\sqrt{2}}\left(\begin{array}{c}
0 \\
v_{0}
\end{array}\right) \quad \text { and } \quad\langle S\rangle=x_{0}
$$

for the SM Higgs doublet which gives rise to the electroweak symmetry breaking and for the singlet scalar Higgs, respectively. These quantities are written with respect to the parameters introduced in the Lagrangian by the following relations derived from the extremum conditions, $\left.\frac{\partial V}{\partial H}\right|_{\langle H\rangle=\frac{v_{0}}{\sqrt{2}}}=0$ and $\left.\frac{\partial V}{\partial S}\right|_{\langle S\rangle=x_{0}}=0$ :

$$
\begin{aligned}
& \mu^{2}=\lambda_{0} v_{0}^{2}+\left(\lambda_{1}+\lambda_{2} x_{0}\right) x_{0} \\
& m_{0}^{2}=-\frac{\lambda_{3}}{2} x_{0}-\frac{\lambda_{4}}{6} x_{0}^{2}-\frac{\lambda_{1} v_{0}^{2}}{2 x_{0}}-\lambda_{2} v_{0}^{2} .
\end{aligned}
$$

We define the scalar fields $h$ and $s$ as the deviation from the vacuum expectation values of $H$ and $S$, respectively. As it is obvious these fields are not mass eigenstates. The 
various components of the mass matrix are written as follows:

$$
\begin{aligned}
& \left.\mu_{h}^{2} \equiv \frac{\partial^{2} V}{\partial h^{2}}\right|_{h=s=0}=2 \lambda_{0} v_{0}^{2} \\
& \left.\mu_{s}^{2} \equiv \frac{\partial^{2} V}{\partial s^{2}}\right|_{h=s=0}=\frac{\lambda_{3}}{2} x_{0}+\frac{\lambda_{4}}{3} x_{0}^{2}-\frac{\lambda_{1} v_{0}^{2}}{2 x_{0}} \\
& \left.\mu_{h s}^{2} \equiv \frac{\partial^{2} V}{\partial h \partial s}\right|_{h=s=0}=\left(\lambda_{1}+2 \lambda_{2} x_{0}\right) v_{0} .
\end{aligned}
$$

The mass eigenstates $h_{1}$ and $h_{2}$ are

$$
\begin{gathered}
h_{1}=\sin (\theta) s+\cos (\theta) h, \\
h_{2}=\cos (\theta) s-\sin (\theta) h,
\end{gathered}
$$

where the mixing angle $\theta$ is defined by

$$
\tan (\theta) \equiv \frac{y}{1+\sqrt{1+y^{2}}},
$$

with $y=\frac{2 \mu_{h s}^{2}}{\left(\mu_{h}^{2}-\mu_{s}^{2}\right)}$. The Higgs boson masses $m_{1}$ and $m_{2}$ are given by

$$
m_{1,2}^{2}=\frac{\mu_{h}^{2}+\mu_{s}^{2}}{2} \pm \frac{\mu_{h}^{2}-\mu_{s}^{2}}{2} \sqrt{1+y^{2}}
$$

This extension of the SM introduces some new parameters. In the sense of experiment, there exist allowed regions in the parameters space where it is possible to explain the relic abundance of the singlet fermions so that they serve as cold dark matter [6]. Before the study of monochromatic gamma ray, we give a reanalysis of the relevant parameters. At the tree level of perturbation, the annihilation of the singlet fermions 
into the standard model particles is given by [6]

$$
\begin{aligned}
\sigma v_{r e l}= & \frac{\left(g_{S} \sin \theta \cos \theta\right)^{2}}{16 \pi}\left(1-\frac{4 m_{\psi}^{2}}{s}\right) \\
\times & \left(\frac{1}{\left(s-m_{h_{1}}^{2}\right)^{2}+m_{h_{1}}^{2} \Gamma_{h_{1}}^{2}}+\frac{1}{\left(s-m_{h_{2}}^{2}\right)^{2}+m_{h_{2}}^{2} \Gamma_{h_{2}}^{2}}\right. \\
& \left.\quad+\frac{2\left(s-m_{h_{1}}^{2}\right)\left(s-m_{h_{2}}^{2}\right)+2 m_{h_{1}} m_{h_{2}} \Gamma_{h_{1}} \Gamma_{h_{2}}}{\left(\left(s-m_{h_{1}}^{2}\right)^{2}+m_{h_{1}}^{2} \Gamma_{h_{1}}^{2}\right)\left(\left(s-m_{h_{2}}^{2}\right)^{2}+m_{h_{2}}^{2} \Gamma_{h_{2}}^{2}\right)}\right) \\
\times & {\left[\left(\frac{m_{b}}{v_{0}}\right)^{2} \cdot 2 s\left(1-\frac{4 m_{b}^{2}}{s}\right)^{3 / 2} \cdot 3+\left(\frac{m_{t}}{v_{0}}\right)^{2} \cdot 2 s\left(1-\frac{4 m_{t}^{2}}{s}\right)^{3 / 2} \cdot 3\right.} \\
& +\left(2 \frac{m_{W}^{2}}{v_{0}}\right)^{2}\left(2+\frac{\left(s-2 m_{W}^{2}\right)^{2}}{4 m_{W}^{4}}\right) \cdot \sqrt{1-\frac{4 m_{W}^{2}}{s}} \\
& \left.+\left(2 \frac{m_{Z}^{2}}{v_{0}}\right)^{2}\left(2+\frac{\left(s-2 m_{Z}^{2}\right)^{2}}{4 m_{Z}^{4}}\right) \cdot \sqrt{1-\frac{4 m_{Z}^{2}}{s}} \cdot \frac{1}{2}\right] \\
+ & \sum_{i, j=1,2} \sigma_{h_{i} h_{j}}+\sum_{i, j, k=1,2} \sigma_{h_{i} h_{j} h_{k}},
\end{aligned}
$$

where $\Gamma_{h_{i}}$ is the decay width of $h_{i}$ for $i=1,2$, and $\sigma_{h_{i} h_{j}}, \sigma_{h_{i} h_{j} h_{k}}$ are the annihilation cross sections of $\bar{\psi} \psi$ into $h_{i} h_{j}$ or $h_{i} h_{j} h_{k}$ with $i, j, k=1,2$. Now we can compute the thermal average of the cross section over $s,\langle\sigma v\rangle$, which is given by [17]

$$
\langle\sigma v\rangle=\frac{1}{8 m^{4} T_{F} K_{2}^{2}\left(m / T_{F}\right)} \int_{4 m^{2}}^{\infty} d s \sigma(s)\left(s-4 m^{2}\right) \sqrt{s} K_{1}\left(\frac{\sqrt{s}}{T_{F}}\right),
$$

where $K_{n}(x)$ is the modified Bessel function of order $n$. The freeze-out temperature $T_{F}$ is estimated by the following iterative equation:

$$
x_{F}=\ln \left(\frac{m}{2 \pi^{3}} \sqrt{\frac{45 M_{\mathrm{pl}}}{2 g_{*} x_{F}}}\langle\sigma v\rangle\right) .
$$

where $x_{F}=m / T_{F}$ and $M_{\mathrm{pl}}=1.22 \times 10^{19} \mathrm{GeV}$ is the Plank mass and $g_{*}=91.5[18]$.

We consider the SFDM with mass below $200 \mathrm{GeV}$ which is the range of Fermi-Lat data in hand [14]. Moreover, we take the SM Higgs mass $125 \mathrm{GeV}$ which is consistent with the new reported value by ATLAS and CMS, and $500 \mathrm{GeV}$ for the other Higgs. Therefore, the annihilation of SFDMs into Higgs particles is not possible. There are a couple of free parameters in the thermally averaged annihilation cross section, in addition to the dark matter mass and Higgs masses: the coupling between the singlet fermion and singlet Higgs, $g_{s}$, the Higgses mixing parameter $\theta$, and the coupling constants between singlet Higgs and the SM Higgs, $\lambda_{1}$ and $\lambda_{2}$. The dependency of $\lambda_{1}$ and 


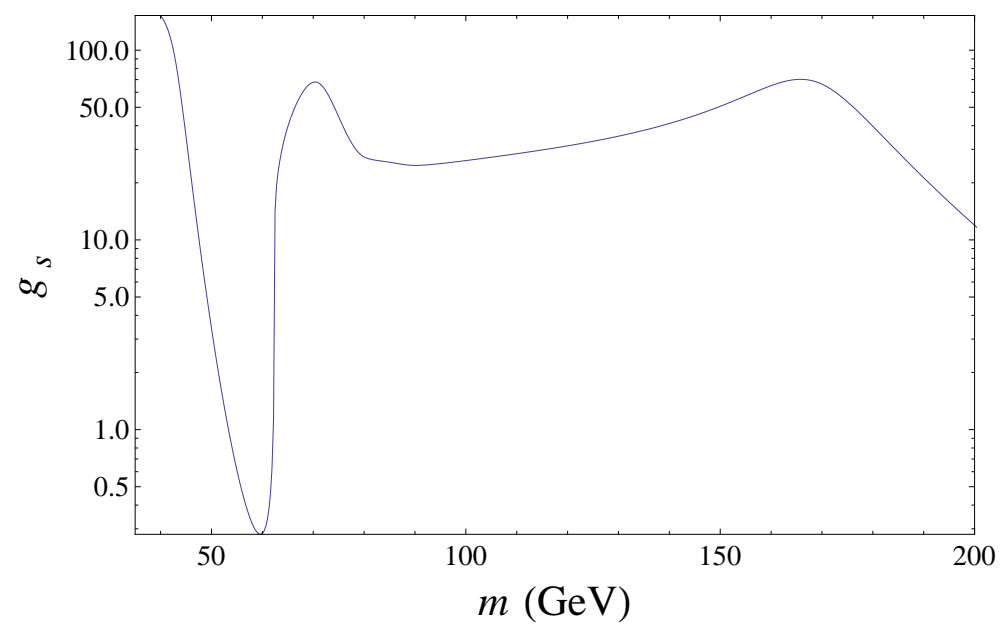

Figure 1: The figure shows the dependency of $g_{s}$ to $m$ with respect to the relic density condition.

$\lambda_{2}$ occurs through the decay width of the Higgs messenger to the lighter Higgs in denominator, so in our desired range of energy these coupling constants are not relevant. This decay rate is also dependent on the mixing angle which is fixed in our calculation. Demanding the correct relic abundance for the dark matter we derive $g_{s}$ in terms of $m$. Since in our calculations the cross sections directly depend on $g_{s} \sin (2 \theta)$, we have fixed $\theta$ to conclude $g_{s}$ (One should note that the effect of the appearance $\theta$ in the Higgs decay width in the denominator is not very significant). Fig. 1 illustrates $g_{s}$ vs dark matter mass with $\sin \theta=0.02$, for instance. It is clear that, if one considers a stronger mixing then the values of $g_{s}$ should be smaller. It is notable that, the valley represents the first resonance region where the dark matter mass is about half of the standard model Higgs mass. In addition, the decreasing region begins at about $170 \mathrm{GeV}$ where the threshold of top quark production is.

\section{The $\bar{\psi} \psi \rightarrow \gamma \gamma$ cross section}

Now, according to the model presented in the previous section, we derive the cross section for the annihilation of two dark matter particles into gamma ray pairs. Although our dark matter cannot directly couple to the SM particles, the annihilation can occur by a Higgs particle through s-channel. In addition, since photon is a massless particle, it does not couple to the Higgs boson directly. Nevertheless, $\mathrm{H} \gamma \gamma$ vertex, can be generated at the quantum level with loops involving massive particles, such as $\mathrm{W}^{ \pm}$bosons and charged fermions. The dominant Feynman diagrams contributing to this process are 
shown in Fig. 2.
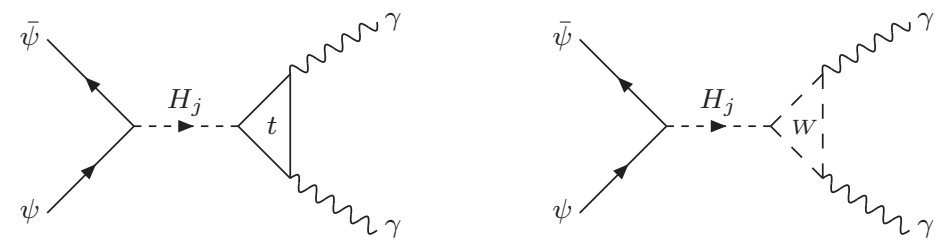

Figure 2: The dominant Feynman diagrams for the annihilation of two singlet fermionic dark matter particles into monochromatic gamma ray lines.

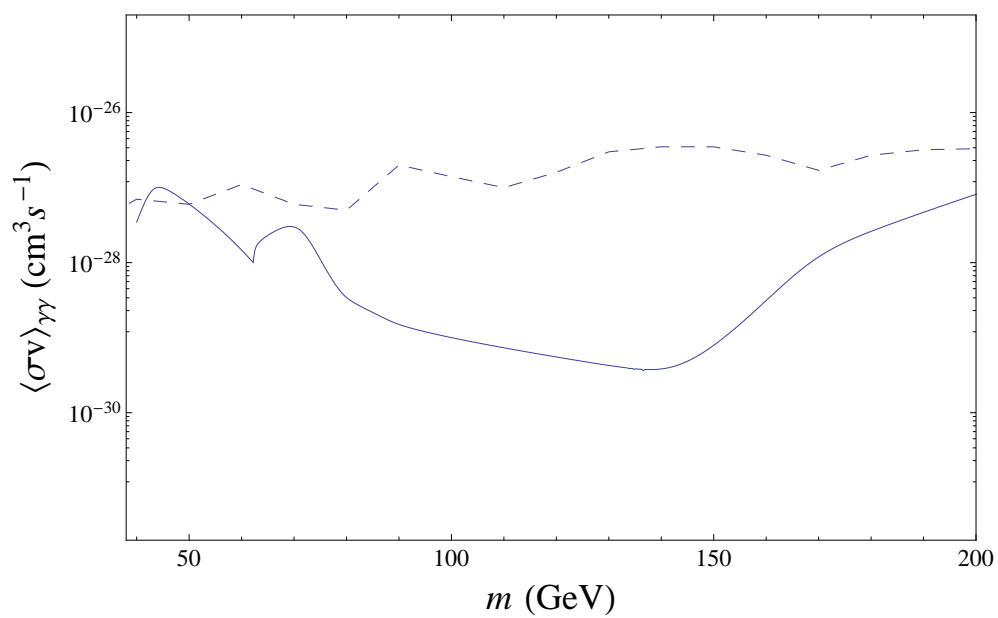

Figure 3: Annihilation cross section into gamma ray lines as a function of dark matter mass for $m_{h_{1}}=125 \mathrm{GeV}, m_{h_{2}}=500 \mathrm{GeV}$, and $g_{s}$ coming from Fig. 1. Dashed line denotes the Fermi-Lat bound [14].

Since the final products are massless, we can write down the cross section as follows:

$$
\sigma v=\frac{1}{8 \pi s} \frac{1}{4} \sum_{\text {spins }}\left|M_{\bar{\psi} \psi \rightarrow \gamma \gamma}\right|^{2}
$$

where $\sqrt{s}$ is the energy in the center of mass frame, and

$$
M_{\bar{\psi} \psi \rightarrow \gamma \gamma}=\sum_{j=1,2} \bar{v}(p)\left(i g_{s} S_{j} \theta\right) u(p) \frac{i}{s-m_{h_{j}}^{2}-i m_{h_{j}} \Gamma_{j}} M_{h_{j} \rightarrow \gamma \gamma}
$$

where $S_{1} \theta$ and $S_{2} \theta$ denote $\sin \theta$ and $\cos \theta$, respectively, and $M_{h_{j} \rightarrow \gamma \gamma}$ is the amplitude for the decay of a Higgs into two photons. Here, $\Gamma_{j}$ is the decay width of $j$ 'th Higgs. One can write $M_{h_{j} \rightarrow \gamma \gamma}$ as follows $[15,16]$ :

$$
M_{h_{j} \rightarrow \gamma \gamma}=\frac{\sqrt{1-\left(S_{i} \theta\right)^{2}} \alpha g s}{8 \pi M_{W}}\left[3\left(\frac{2}{3}\right)^{2} F_{t}+F_{W}\right],
$$


where

$$
\begin{gathered}
F_{t}=-2 \tau[1+(1-\tau) f(\tau)], \\
F_{W}=2+3 \tau+3 \tau(2-\tau) f(\tau) .
\end{gathered}
$$

Here, $\tau=4 m_{i}{ }^{2} / s$ with $i=t, W$ and

$$
f(\tau)= \begin{cases}\left(\sin ^{-1} \sqrt{1 / \tau}\right)^{2}, & \text { for } \quad \tau \geq 1 \\ -\frac{1}{4}\left(\ln \frac{1+\sqrt{1-\tau}}{1-\sqrt{1-\tau}}-i \pi\right)^{2} & \text { for } \quad \tau<1\end{cases}
$$

Therefore, we obtain the cross section as follows:

$$
\begin{gathered}
\sigma v=\frac{1}{8 \pi s} \frac{1}{4} \sum_{\text {spins }}\left|M_{\bar{\psi} \psi \rightarrow \gamma \gamma}\right|^{2}=\frac{1}{32 \pi s} g_{s}{ }^{2}\left(s-4 m^{2}\right)\left\{\frac{\left|M_{h_{1} \rightarrow \gamma \gamma}\right|^{2} \sin ^{2} \theta}{\left(m_{h_{1}}{ }^{2}-s\right)^{2}+m_{h_{1}}{ }^{2} \Gamma_{1}{ }^{2}}+\frac{\left|M_{h_{2} \rightarrow \gamma \gamma}\right|^{2} \cos ^{2} \theta}{\left(m_{h_{2}}{ }^{2}-s\right)^{2}+m_{h_{2}}{ }^{2} \Gamma_{2}{ }^{2}}\right. \\
\left.+\frac{\left(s-m_{h_{1}}{ }^{2}\right)\left(s-m_{h_{2}}{ }^{2}\right)+\left|M_{h_{1} \rightarrow \gamma \gamma}\right|\left|M_{h_{2} \rightarrow \gamma \gamma}\right|}{\left[\left(m_{h_{1}}{ }^{2}-s\right)^{2}+m_{h_{1}}{ }^{2} \Gamma_{1}{ }^{2}\right]\left[\left(m_{h_{2}}{ }^{2}-s\right)^{2}+m_{h_{2}}{ }^{2} \Gamma_{2}{ }^{2}\right]} \sin \theta \cos \theta\left(M_{h_{1} \rightarrow \gamma \gamma} M_{h_{2} \rightarrow \gamma \gamma}^{*}+M_{h_{2} \rightarrow \gamma \gamma}^{*} M_{h_{1} \rightarrow \gamma \gamma}\right)\right\} .
\end{gathered}
$$

Thermal average of this cross section is also obtained using (12) and (13). We have illustrated the phenomenological aspects of this calculation via the Fig. 3. The SFDM can be served as cold dark matter provided that the free parameters are chosen such that the relic abundance condition is respected. Therefore, this figure shows cross section versus the dark matter mass while we use $g_{s}$ from Fig. 1. For comparison, we have also shown the upper limits obtained by Fermi-Lat [14] for Navarro-Frenk-White (NFW) density profile. It is noticeable that most of the region (except the resonance) is actually excluded by direct detection experiments, following the results of Figure 6 in Ref. [6]. However we should note that, for masses below about $70 \mathrm{GeV}$ (and above about 150 $\mathrm{GeV}$ ), in particular the resonance regions where are not excluded by direct detection experiment, the cross section is comparable with the Fermi-Lat data. Therefore, these values may be explored by likely future more precise experiments.

\section{Summary and discussion}

In this paper we have considered an extension of the SM which includes a singlet fermion as a cold dark matter and a real singlet scalar as a messenger to explain the corresponding thermal relic density [6]. Demanding correct relic abundance, we have derived $g_{s}$ and plotted it in Fig. 1 versus dark matter mass. This figure is consistent with figure 2 in ref. [6]. Only we have fixed the Higgs mixing angle to obtain a definite value for $g_{s}$. Then the cross section of the annihilation of the singlet fermion into two 
monochromatic photons has been obtained. The thermal averaged of the annihilation of this cross section is calculated for a wide range of energies including resonance. We have depicted the thermally averaged of the SFDM annihilation cross section into two photons for a reasonable set of the Higgs masses in Fig. 3. This figure allows one to compare our result with the Fermi-Lat observations. The most of the region (except the resonance) is excluded by direct detection experiments (see figure 6 in ref. [6]). However, the Fermi-Lat data can be an alternative way for exploring the nature of dark matter. As one can see from Fig. (3), in some region (mass below $70 \mathrm{GeV}$ and above 150 $\mathrm{GeV}$ which include the resonance regions), the two-photon annihilation cross section of SFDM is comparable to the resent Fermi-Lat data and potentially can be explored by likely future more precise experiments.

Acknowledgement: The financial support of the University of Qom research council is acknowledged.

\section{References}

[1] G. Jungman, M. Kamionkowski, and K. Griets, Phys. Rep. 267, 195 (1996).

[2] G. Bertone, D. Hooper, and J. Silk, Phys. Rep. 405, 279 (2005).

[3] H.-C. Cheng, J. L. Feng, and K. T. Matchev, Phys. Rev. Lett. 89, 211301 (2002); G. Servant and T. Tait, Nucl. Phys. B 650, 391 (2003).

[4] H.-C. Cheng and I. Low, J. High Energy Phys. 09 (2003) 051; J. High Energy Phys. 08, 061 (2004).

[5] J. McDonald, Phys. Rev. D 50, 3637 (1994); Phys. Rev. Lett. 88, 091304 (2002); M. C. Bento, O. Bertolami, and R. Rosenfeld, Phys. Lett. B 518, 276 (2001); H. Davoudiasl, R. Kitano, T. Li, and H. Murayama, Phys. Lett. B 609, 117 (2005); C. P. Burgess, M. Pospelov, and T. ter Veldhuis, Nucl. Phys. B 619, 709 (2001).

[6] K.Y. Lee, Y. G. Kim, and S. Shin, J. High Energy Phys. 05, 100 (2008).

[7] Y. G. Kim and K.Y. Lee, Phys. Rev. D 75, 115012 (2007).

[8] M. M. Ettefaghi, Phys. Rev. D79, 065022 (2009).

[9] E. A. Baltz, M. Battaglia, M. E. Peskin, and T. Wizansky, Phys. Rev. D 74, 103521 (2006). 
[10] M. W. Goodman, and E. Witten, Phys. Rev. D31, 3059 (1985); S. Khali and C. Munoz, Contemporary Physics 43, 51 (2002); C. Munoz, Int. J. Mod. Phys. A 19 3093 (2004).

[11] Gianfranco Bertone, [arxiv:astro-ph/0607706].

[12] M. Gustafsson, E. Lundstrom, L. Bergstrom, and J. Edsjo, Phys. Rev. Lett. 99, 041301 (2007) [arxiv:astro-ph/0703512]; C. E. Yaguna, JCAP 0903, 003 (2009) [arxiv:astro-ph/0810.4267];F. Takayama and M. Yamaguchi, Phys. Lett. B485, 388 (2000)[arXiv:hep- ph/0005214];W. Buchmuller, L. Covi, K. Hamaguchi, A. Ibarra and T. Yanagida, JHEP 03, 037 (2007) [arXiv:hep-ph/0702184]; G. Bertone, W. Buchmuller, L. Covi and A. Ibarra, JCAP 11, 003 (2007) [arXiv:0709.2299 [astroph]]; A. Ibarra and D. Tran, Phys. Rev. Lett. 100, 061301 (2008) [arXiv:0709.4593 [astro-ph]]; K. Ishiwata, S. Matsumoto and T. Moroi, Phys. Rev. D78; 063505 (2008) [arXiv:0805.1133 [hep-ph]]; W. Buchmuller, A. Ibarra, T. Shindou, F. Takayama and D. Tran, JCAP 09, 021 (2009)[arXiv:0906.1187 [hep-ph]]; K. Y. Choi, D. E. Lopez-Fogliani, C. Munnoz and R. R. de Aus- tri, JCAP 03, 028 (2010) [arXiv:0906.3681 [hep-ph]]; D. Restrepo, M. Taoso, J.W.F. Valle, O. Zapata, Phys. Rev. D85, 023523 (2012) [arXiv:1109.0512[hep- ph]]; G.A. Gomez-Vargas et al., JCAP 02021 (2012) [arXiv:1110.3305 [astro- ph]]; F. Boudjema, A. Semenov, D. Temes, Phys. Rev. D72, 055024 (2005); C. Boehm, J. Orloff, P. Salati, Phys. Lett. B641, 247 (2006); L. Bergstrom, T. Bringmann, M. Eriksson, M. Gustafsson, JCAP 0504, 004 (2005); etc..

[13]

Y. G. Kim, S. Shin, JHEP 0905, 036 (2009); S. Baek, P. Ko, W. Park, [arxiv:hep$\mathrm{ph} / 1112.1847]$.

[14] A.A. Abdo et al., Phys. Rev. Lett. 104091302 (2010) [arXiv:1001.4836].

[15] A.I. Vainshtein, M.B. Voloshin, V.I. Sakharov and M.A. Shifman, Yad. Fiz. 30 (1979) 1368 [Sov. J. Nucl. Phys. 30 (1979) 711].

[16] J.F. Gunion, G.L. Kane, H. E. Haber, and S. Dawson, The Higgs Hunter's Guide (Addison-Wesley, Reading, MA, 1990).

[17] P. Gondolo and G. Gelmini, Nucl. Phys. B360, 145 (1991).

[18] E.W. Kolb and M.S. Turner, The Early Universe (Addison Wesley, New York, 1990) 
[19] A. A. Abdo et al. [Fermi-LAT Collaboration], JCAP 1004, 014 (2010) [arXiv:1002.4415 [astro-ph.CO]]

[20] M. Gustafsson, E. Lundstrom, L. Bergstrom and J. Edsjo, Phys. Rev. Lett. 99 041301 (2007) [arXiv:astro-ph/0703512]. 